\title{
The Chromospheric Response to the Sunquake Generated by the X9.3 Flare of NOAA 12673
}

\author{
Sean Quinn $^{1}$ (D), Aaron Reid ${ }^{1}$ (D), Mihalis Mathioudakis ${ }^{1}$ (D), Christoper Nelson ${ }^{1}$, S. Krishna Prasad ${ }^{1}$ (D), and Sergei Zharkov ${ }^{2}$ (D) \\ ${ }^{1}$ Astrophysics Research Centre, School of Mathematics and Physics, Queen's University Belfast, BT7 1NN, UK \\ ${ }^{2}$ E.A. Milne Centre for Astrophysics, School of Mathematics and Physical Sciences, Hull University, Kingston upon Hull, HU6 7RX, UK \\ Received 2019 April 18; revised 2019 June 6; accepted 2019 June 19; published 2019 August 14
}

\begin{abstract}
Active region NOAA 12673 was extremely volatile in 2017 September, producing many solar flares, including the largest of solar cycle 24, an X9.3 flare of 2017 September 06. It has been reported that this flare produced a number of sunquakes along the flare ribbon. We have used cotemporal and cospatial Helioseismic and Magnetic Imager (HMI) line of sight (LOS) and Swedish $1 \mathrm{~m}$ Solar Telescope (SST) observations to show evidence of the chromospheric response to these sunquakes. Analysis of the Ca II $8542 \AA$ line profiles of the wavefronts revealed that the crests produced a strong blue asymmetry, whereas the troughs produced at most a very slight red asymmetry. We used the combined HMI, SST data sets to create time-distance diagrams and derive the apparent transverse velocity and acceleration of the response. These velocities ranged from 4.5 to $29.5 \mathrm{~km} \mathrm{~s}^{-1}$ with a constant acceleration of $8.6 \times 10^{-3} \mathrm{~km} \mathrm{~s}^{-2}$. We employed NICOLE inversions, in addition to the center-of-gravity method to derive LOS velocities ranging from $2.4 \mathrm{~km} \mathrm{~s}^{-1}-3.2 \mathrm{~km} \mathrm{~s}^{-1}$. Both techniques show that the crests are created by upflows. We believe that this is the first chromospheric signature of a flare induced sunquake.
\end{abstract}

Key words: Sun: chromosphere - Sun: flares - Sun: helioseismology

Supporting material: animation

\section{Introduction}

Solar flares involve the impulsive release of energy throughout the solar atmosphere. They are associated with active regions (ARs) with large polarity inversion lines where the complexity of the magnetic field gives rise to reconnection in the corona. The antiparallel movement of charged plasma can lead to the formation of a "current sheet" and the instability created causes opposing filaments to reconnect. The plasma above the reconnection site can be accelerated into interplanetary space while the plasma below moves down the filaments and toward the solar surface. It is this downward moving plasma that can disturb the lower atmosphere. The suggestion that solar flares can generate seismic response was first put forward by Wolff (1972) and was more recently explored by Kosovichev \& Zharkova (1995). The first detection of flare induced sunquakes (SQs) was reported in 1998 (Kosovichev \& Zharkova 1998), using the Michelson Doppler Interferometer (Scherrer et al. 1995) on board the Solar and Heliospheric Observatory (Domingo et al. 1995). The SQs appeared as expanding circular waves in photospheric Dopplegrams that were created during the impulsive phase of the flare event.

The widely accepted explanation for the generation of the SQ is associated with the "collisional thick-target" model (CTTM), where an SQ is produced by acoustic waves that travel into the solar interior where they are refracted by the denser plasma. In this model, a beam of high-energy particles is accelerated from the reconnection site toward the deeper layers of the solar atmosphere where it deposits large amounts of energy and momentum (Kosovichev 2007). The collisions of the accelerated particles with the chromospheric plasma result into heating and the production of hard and soft X-ray (HXR and SXR) emission. The heated plasma expands, causing a compression known as a "chromospheric condensation," which travels deeper into the solar atmosphere and collides with the denser photosphere. The collision creates a high pressure compression in the photosphere, causing a downward propagating shock front (Kosovichev 2006). The shock imparts energy through the convection zone, where it is reflected due to changes in density and temperature, and appears as expanding ripples on the solar surface.

Alternative sources of energy deposition into the photosphere have also been proposed. These include photospheric heating due to continuum radiation (Lindsey \& Donea 2008), or deeply penetrating proton beams (Zharkova \& Zharkov 2007). These models can explain seismic events where HXR or white light emission is detected and provide an explanation for SQ generation. One of the issues with these interpretations is that the shock front that propagates in the solar interior can experience significant damping, depleting the energy of the seismic wave (Russell et al. 2016). Furthermore, the source of the SQ is sometimes detected away from the site of the HXR emission (Zharkov et al. 2011). This suggests that the SQ is not uniquely formed at the site of the accelerated particle collisions. The SQ ripples are often observed during the impulsive phase of the flare, before the maximum HXR and SXR emission (Zharkov et al. 2011).

While SQs are sometimes observable using Dopplergrams alone, time-distance diagrams can also be employed for the analysis of the observed ridges (Kosovichev \& Zharkova 1998; Zharkova \& Zharkov 2007; Sharykin \& Kosovichev 2018; Zhao \& Chen 2018). Once the time-distance diagram is created, a theoretical regression trend can normally be fitted to the data, with a match to any SQ ridge that is present in the diagram. The regression trend uses the ray-path approximation (Couvidat et al. 2004) with a more detailed description provided by Kosovichev (2011).

Further analysis can be conducted by implementing regression techniques such as acoustic holography (Donea \& Lindsey 2005; Donea 2011). Acoustic holography can be used 
to reveal the source of the SQ by creating a partial reconstruction of the acoustic field using Dopplergrams of the flaring region. These images are averaged over their cadence and convolved with a Green's function, in terms of height in the atmosphere, as well as horizontal distance from an approximate source and time (Donea et al. 1999). The Green's function allows the observed ripple in the Dopplergrams to be traced back to a point source (Matthews et al. 2011, 2015). The convolution of these two functions creates a regression map. The regression power map displays sources of acoustic emission as bright kernels, and sources of acoustic absorption as dark kernels (Donea et al. 1999). The map can be filtered in frequency to gain information on the formation height of the sources and sinks.

Despite the increased number of SQ detections provided by the Solar Dynamics Observatory (SDO; Pesnell et al. 2012) their relationship with the flare energy released remains unclear. For example, a number of X-class flares have been reported without SQs, where low M-class flares have been reported as having associated SQs (Kosovichev 2014). This is not to say that there is no seismic response present as it may lie below the solar background noise. Analysis of the atmospheric response to SQs, such as time delays and intensity variations, can provide insights on the temperature gradients and intensity variations in the solar interior. It has been predicted that a global seismic response can also be excited by a solar flare. Such seismic responses would have a very low amplitude, below the amplitude of random solar oscillations, hence detection has been proven difficult (Kosovichev 2014).

In this article, we present evidence of a chromospheric response to a sunquake following the X9.3 solar flare of 2017 September 6, the largest flare of Solar Cycle 24. The flare originated from AR NOAA 12673 and the GOES flux peaked at approximately 11:55:00 UT. Photospheric disturbances associated with this flare have been reported in the literature (Sharykin \& Kosovichev 2018; Zhao \& Chen 2018). We apply time-distance analysis to derive the apparent transverse velocity and acceleration of the SQ. The corresponding LOS velocity is derived with the center-of-gravity (COG) method and inversion techniques.

\section{Observations}

The ground-based data analyzed here were collected between 11:57:17 UT and 12:52:05 UT on 2017 September 6 by the CRisp Imaging SpectroPolarimeter (CRISP; Scharmer et al. 2008) attached to the Swedish Solar Telescope (SST; Scharmer et al. 2003). During this time, CRISP was pointed at coordinates of $X=537^{\prime \prime}, Y=-222^{\prime \prime}(\mu=0.79)$ and ran a sequence sampling the $\mathrm{Ca}$ II $8542 \AA$ and $\mathrm{H} \alpha$ lines, with the Ca II $8542 \AA$ data being observed in full-Stokes polarimetry mode. The Ca II $8542 \AA$ scan included 11 line positions at $\pm 0.7 \AA, \quad \pm 0.5 \AA, \pm 0.3 \AA, \pm 0.2 \AA, \pm 0.1 \AA$, as well as the line core. The $\mathrm{H} \alpha$ scan included 13 line positions, at $\pm 1.5 \AA$, $\pm 1.0 \AA, \pm 0.8 \AA, \pm 0.6 \AA, \pm 0.3 \AA, \pm 0.15 \AA$, and the line core. Wide-band (WB) images were also obtained cotemporal with each CRISP narrowband image for alignment purposes.

These SST data were processed using the Multi-object MutiFrame Blind Deconvolution (Löfdahl 2002; Van Noort et al. 2005) method. This included the subdivision of each image into $88 \times 88$ pixel $^{2}$ subimages, each of which was individually restored to maintain the presumed invariance of the image formation modules. A prefilter field of view (FOV) and wavelength dependent correction was applied to each restored image. More information on the SST reduction pipeline is provided in de la Cruz Rodríguez et al. (2014). The analysis detailed in this article was conducted on a reduced CRISP FOV of 47 !' $0 \times 47$ !" 0 in order to avoid fringing effects at the edge of the CCDs. These data have a pixel scale of 0 " 059 and cadence of $15 \mathrm{~s}$.

In addition to CRISP, we also analyze data sampled by the $S D O$ 's Helioseismic and Magnetic Imager (SDO/HMI; Scherrer et al. 2012) and Atmospheric Imaging Assembly (SDO/AIA; Pesnell et al. 2012). HMI obtains full disk line-ofsight (LOS) velocity and magnetic field measurements, as well as photospheric continuum images, every $45 \mathrm{~s}$ with a pixel scale of 0 ". 5 . A detailed explanation on the HMI LOS velocity maps is provided in Schou et al. (2011), Couvidat et al. (2011). The HMI LOS velocity data best displayed the presence of an $\mathrm{SQ}$, and was chosen to be the photospheric representation for this analysis. It should be noted, though, that the HMI continuum and magnitude data cubes also showed some seismic responses.

Only the 1600 and $1700 \AA$ filters from the SDO/AIA instrument were analyzed here. These filters clearly displayed the SQ and had a pixel scale of 0 " 6 and a cadence of $24 \mathrm{~s}$. An extended $240^{\prime \prime} \times 240^{\prime \prime}$ FOV was studied for all SDO data to investigate the propagation of the SQ analyzed out of the limited FOV of the CRISP instrument. The SDO data were coaligned with the SST using routines developed by R. J. Rutten. ${ }^{3}$

\section{Results}

\subsection{Properties of the SQs}

During the evolution of the flare, the Ca II $8542 \AA$ data revealed multiple wavefronts emanating from the flare ribbon. In Figure 1, we plot four temporal snapshots of the flare and associated SQ at a spectral position of $8541.8 \AA$ ( $0.2 \AA$ away from the Ca II $8542 \mathrm{~A}$ line core). This line position was chosen as it showed the clearest signature of the SQs. The two green arrows in the bottom right image indicate the seismic responses, appearing as wavefronts, that emanated from two sources in the flare ribbon. The "upper" source appeared to excite waves propagating westward, while the "lower" source emitted waves moving in a southwesterly direction. We refer to these sources as "upper" and "lower" sources due to their locations in relation to the flare ribbon. The blue and red arrows highlight crests and troughs of the apparent waves, respectively. It should be noted that the wavefronts can be detected at other positions across the Ca II $8542 \AA$ line profile; however, the contrasts between the waves and the background were not as great at these spectral locations as at $8541.8 \AA$.

To better accentuate these seismic responses, we created running difference maps for both the SDO and CRISP data sets. These running difference maps were created using the formula $\operatorname{diff}(n)=\operatorname{frame}(n)-\operatorname{frame}(n-1)$. The chromospheric Ca II $8542 \AA$ seismic response to the flare was first detected in these running difference images at approximately 12:11:33 UT. Although wavefronts could also be detected in a running difference of the $\mathrm{H} \alpha$ line core, the flare ribbon was significantly brighter in these data meaning achieving accurate inferences about the SQ properties proved more difficult. Therefore, the

\footnotetext{
http://www.staff.science.uu.nl/ rutte101/rridl/sdolib/
} 

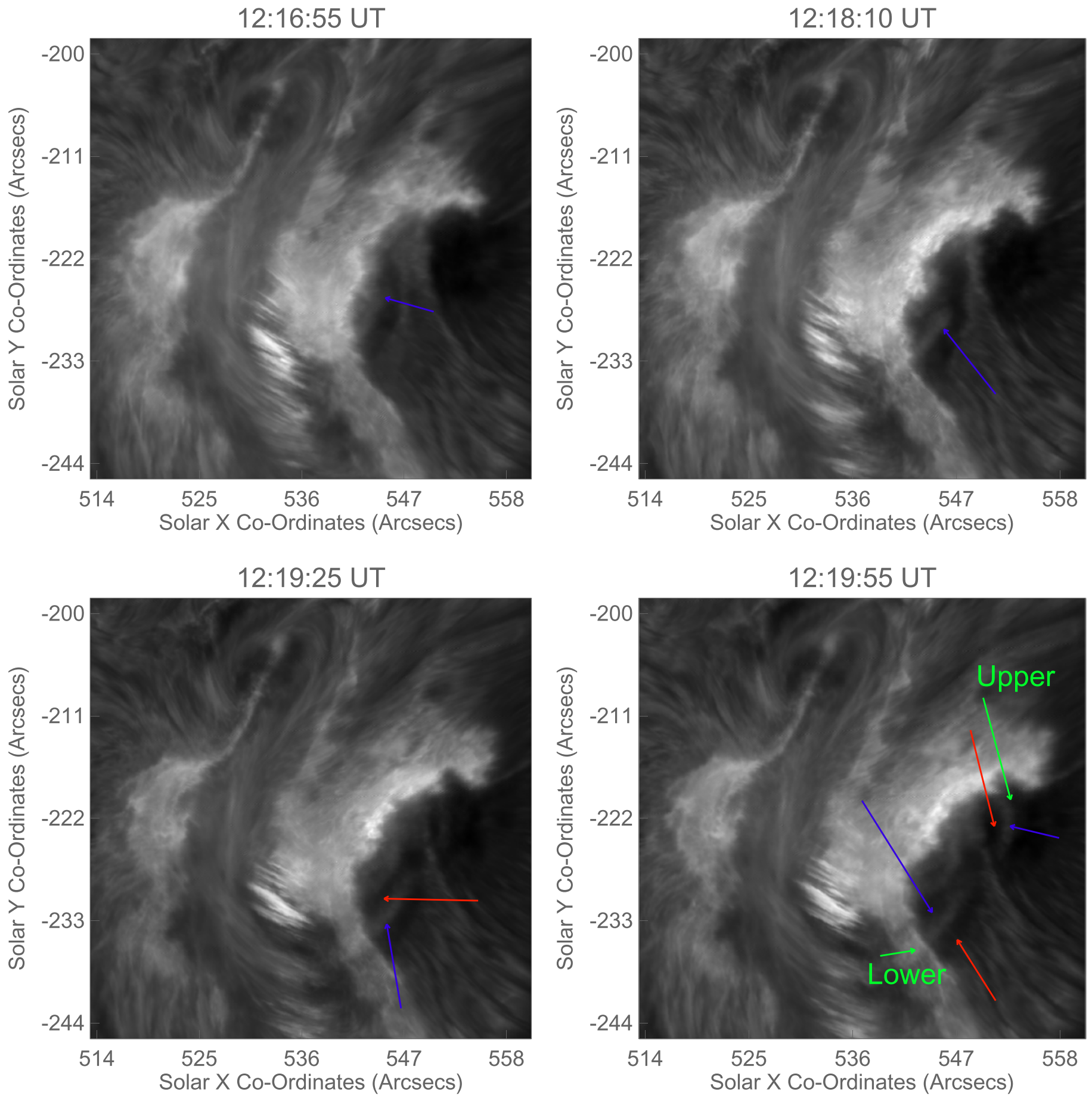

Figure 1. The $47^{\prime \prime} \times 47^{\prime \prime}$ SST FOV analyzed here plotted at four time-steps (indicated by each panel title) at $8541.8 \AA$. The blue arrows point to "crests" (brightenings) and the red arrows point to "troughs" (darkenings) of the chromospheric response to the SQ. The two green arrows in the bottom right panel point to the "upper" and "lower" responses.

majority of this work was undertaken on the Ca II $8542 \AA$ data. Running difference maps from four diagnostics are shown in Figure 2, where the green arrows overlaid on each panel indicate the wavefronts. The red box on the HMI LOS image (top right) depicts the cotemporal SST CRISP FOV plotted in the lower panels. The first seismic response appears in the HMI Dopplegram at approximately 12:12:02 UT from the lower source. As this response was observed to be the longest, it will be the main focus of our subsequent analysis.
Analysis of the coaligned CRISP and HMI LOS data sets revealed that the Ca II $8542 \AA$ seismic wave signatures propagated in the same direction as those detected in HMI (Figure 3). The blue arrow indicates the wave detected in CRISP data at 12:18:37 UT, and the red arrow indicates the HMI LOS wave at 12:22:57 UT. While there are multiple SQs detected in HMI, the wavefront that aligns both spatially and temporally with the chromospheric response first appeared at 12:12:02 UT. The correspondence between the signatures in CRISP and HMI data will be studied in more detail in the 

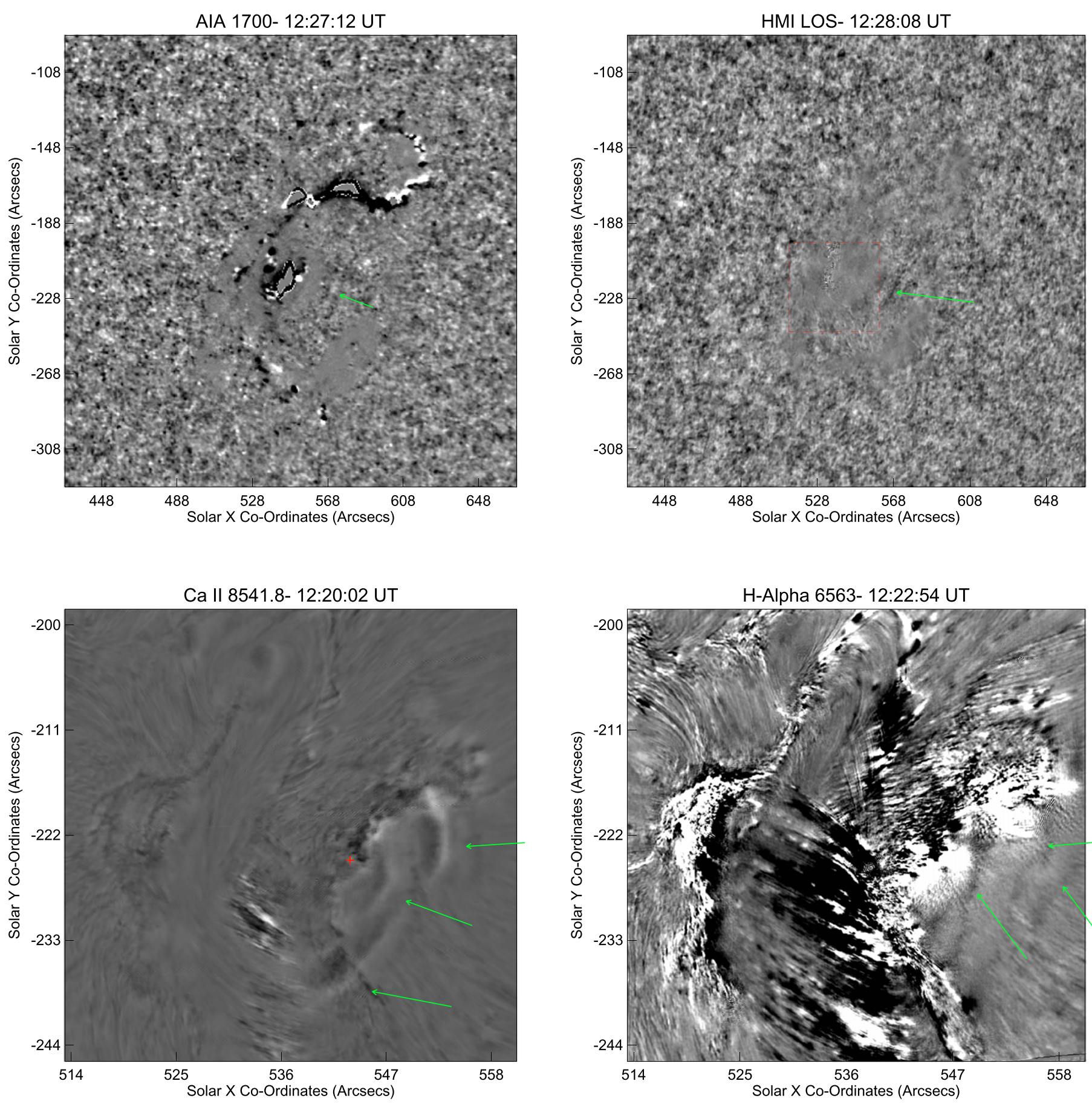

Figure 2. Difference images that show the seismic response of the atmosphere to the X9.3 flare of 2017 September 06 . Top: SDO AIA $1700 \AA$ and the HMI LOS running differences. The dotted red box overlaid on the HMI LOS image indicates the CRISP FOV plotted in the bottom panels. Bottom: cotemporal SST running difference images from Ca II $8541.8 \AA$ and $\mathrm{H} \alpha$. Green arrows indicate the presence of wavefronts. The red cross in the bottom left image is the estimated epicenter of the Ca II $8541.8 \AA$ response.

(An animation of this figure is available.)

following subsection. As the CRISP instrument has a smaller FOV and higher spatial resolution than the HMI, it is not unexpected that the chromospheric signature of the SQ is detected prior to the photospheric signature. The stark intensity contrast in the $\mathrm{H} \alpha$ running difference discussed previously can be seen in the bottom right of Figure 2. Any intensity increase created as a wavefront moves across the image would be lost in the apparent "background noise" created by the contrasting intensities.

\subsection{Time-Distance Analyses}

In order to study the progress of the waves from the lower source, an approximate point-of-origin was identified for these apparent waves and time-distance diagrams were created by selecting a pixel range and angle, over which the wavefront propagated. By tracing back the wavefronts, the origin of the seismic response was approximated as $X=543$." 16 , $Y=-225$ ". 87 , displayed as the red cross in the bottom left 


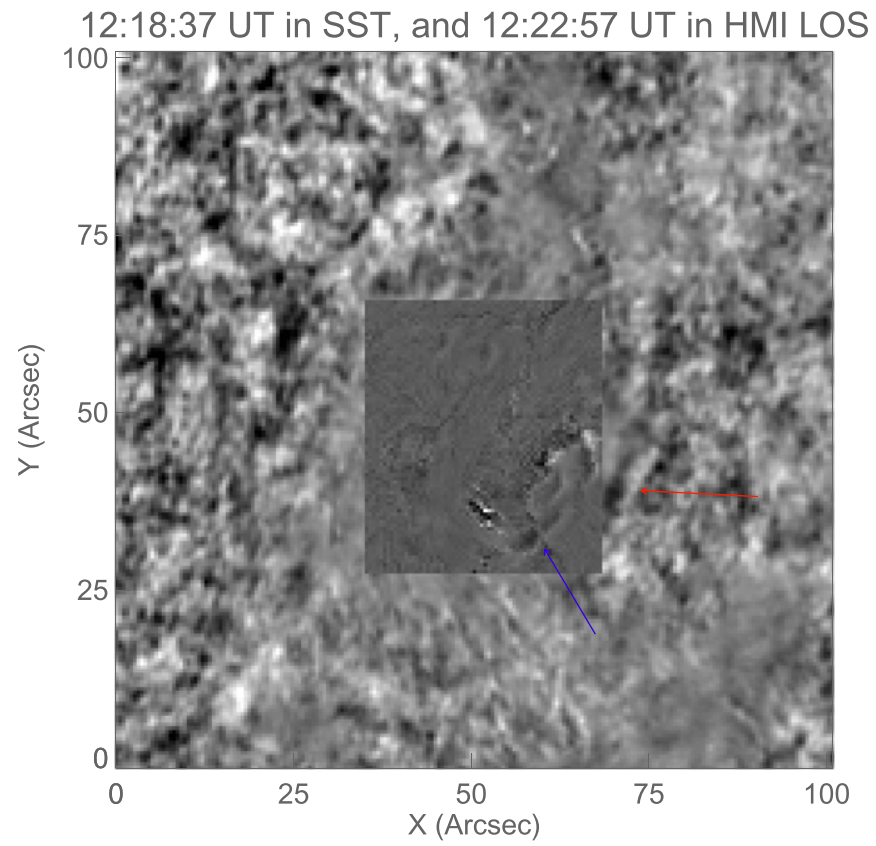

Figure 3. Ca II $8542 \AA$ running difference (overlaid panel) aligned to a HMI LOS running difference image (background panel) showing the progression of the wavefront across the two data sets. The CRISP image was obtained at 12:18:37 UT and the HMI at 12:22:57 UT. The blue and red arrows indicate the seismic response in the chromosphere and photosphere, respectively.

image of Figure 2. Acoustic holography (Donea et al. 1999) techniques were undertaken to better identify the sources of the seismic responses but these proved unsuccessful. This was most likely due to the difference in height at which the holography was undertaken. Normally, acoustic holography is undertaken in the photosphere, whereas ours was in the chromosphere. This difference in height most likely proved the problem, causing the technique to be unsuccessful. The waves were observed to propagate approximately $8.58 \mathrm{Mm}$ from this epicenter and between angles $125^{\circ}$ and $145^{\circ}$, where $0^{\circ}$ is solar north. The left panel of Figure 4 plots the time-distance diagram constructed from the CRISP Ca II 8541.8 $\AA$ data. Pixels where the wavefront was present appeared more intense, hence they created ridges of higher intensity. Pixels in the trough of the wave were lower in intensity creating the striking light-dark streaks. The same procedure was undertaken for the upper response but this was more noisy and had more interference with the flare ribbon.

Multiple ridges can be seen in the CRISP time-distance diagram, indicated by the blue arrows in Figure 4, consistent with the presence of multiple wavefronts in Figure 1. We followed a similar procedure to that conducted by Sharykin \& Kosovichev (2018) and fitted the initial ridge on the CRISP time-distance diagram in Figure 4 with a regression trend of $x^{0.5}$, that SQs commonly possess (Kosovichev \& Zharkova 1998). We selected this ridge as it was not disturbed by the presence of the dynamic flare ribbons indicated with the green arrows in the left-hand panel of Figure 4. This theoretical trend matches well with the observed propagation of the wave and is depicted as the red dashed-dotted line in the left-hand panel of Figure 4 (moved vertically downwards by a few pixels in order that it did not obscure the wavefront).

The observed Ca II $8542 \AA$ wavefronts, which move in an apparent circular arc pattern from two different locations, are consistent with being the chromospheric components of the photospheric SQs analyzed by Sharykin \& Kosovichev (2018). In order to study the propagation of the waves from the CRISP FOV to the HMI FOV, a time-distance diagram that combined the CRISP and HMI data sets was constructed. This is plotted in image B of Figure 4. The HMI data were extrapolated such that their cadence was cotemporal with the CRISP data and the spatial resolution of the CRISP data set was degraded to that of HMI. The same epicenter and angle over which the wavefront propagated through as in image A of Figure 4 were used; however, a longer slit was created in order to study the wave propagation into the surrounding atmosphere. The left side of the CRISP-HMI time-distance diagram (to the left of the green vertical line) shows the "overplotted" CRISP data, with a number of ridges, which correspond to the multiple wavefronts observed in running difference. The times and positions where the wavefront can be identified in the combined CRISP-HMI data set, correlate well with the position of the ridges in the CRISP time-distance diagram indicating that the seismic wave observed in the CRISP data is a chromospheric response to the photospheric SQ reported previously by Sharykin \& Kosovichev (2018).

Using the larger FOV provided by the combined CRISP and HMI data sets, we are able to further analyze the temporal behavior of these seismic responses. The red dots in Figure 4(B) mark the apparent locations of the wavefront through time which are used to model the apparent propagation of the response. A $\chi^{2}$ analysis was then conducted to determine which trend would best model these data points: a linear, a trend of $x^{0.5}$ (as theory would predict), or a second-order polynomial. Our analysis revealed that a function of the shape of the theoretical regression trend, $x^{0.5}$, provided the most accurate fit to both the SST and HMI regions of the timedistance diagram. This trend is plotted by the blue line in image B of Figure 4. As in image A, these points have been shifted slightly downwards to allow the ridge to become more visible.

Tracking the apparent position of the wave through time allows us to estimate its velocity and acceleration. The velocity of the wavefront ranged from $4.5 \mathrm{~km} \mathrm{~s}^{-1}$ at its initial time to $29.5 \mathrm{~km} \mathrm{~s}^{-1}$ as it propagated out of the analyzed FOV. The wavefront gradually accelerated at a rate of $8.6 \times 10^{-3} \mathrm{~km} \mathrm{~s}^{-2}$ and traveled for approximately $117 \mathrm{Mm}$ from the estimated epicenter in 46.5 minutes. This entire propagation was not used in the creation of Figure 4(B), as the ridge becomes nearly indistinguishable from background noise at these distances, while remaining visible by eye in the HMI LOS running difference.

Before the calculation of these wavefront velocities, one may be reminded of another flare initiated chromospheric response, namely, Moreton waves (Moreton \& Ramsey 1960; Chen et al. 2011). These waves propagate across very large distances $\left(5 \times 10^{5} \mathrm{~km}\right)$ at extremely high velocities $\left(500-2000 \mathrm{~km} \mathrm{~s}^{-1}\right.$; Chen et al. 2011). However, these velocities and propagation distances confirm the wavefronts are a representation of a chromospheric component of an SQ.

A number of such wavefronts are apparent in the HMI LOS running difference data; however, only one appears to propagate outside of the SST FOV. We have also investigated the SQ in the SDO/AIA 1600 and $1700 \AA$ channels. Our selection for a region of interest in these channels was guided by HMI and the cadence was adjusted to fit with the HMI running difference. Signatures of the SQ were detected in both AIA channels. An AIA $1700 \AA$ A running difference map is also 

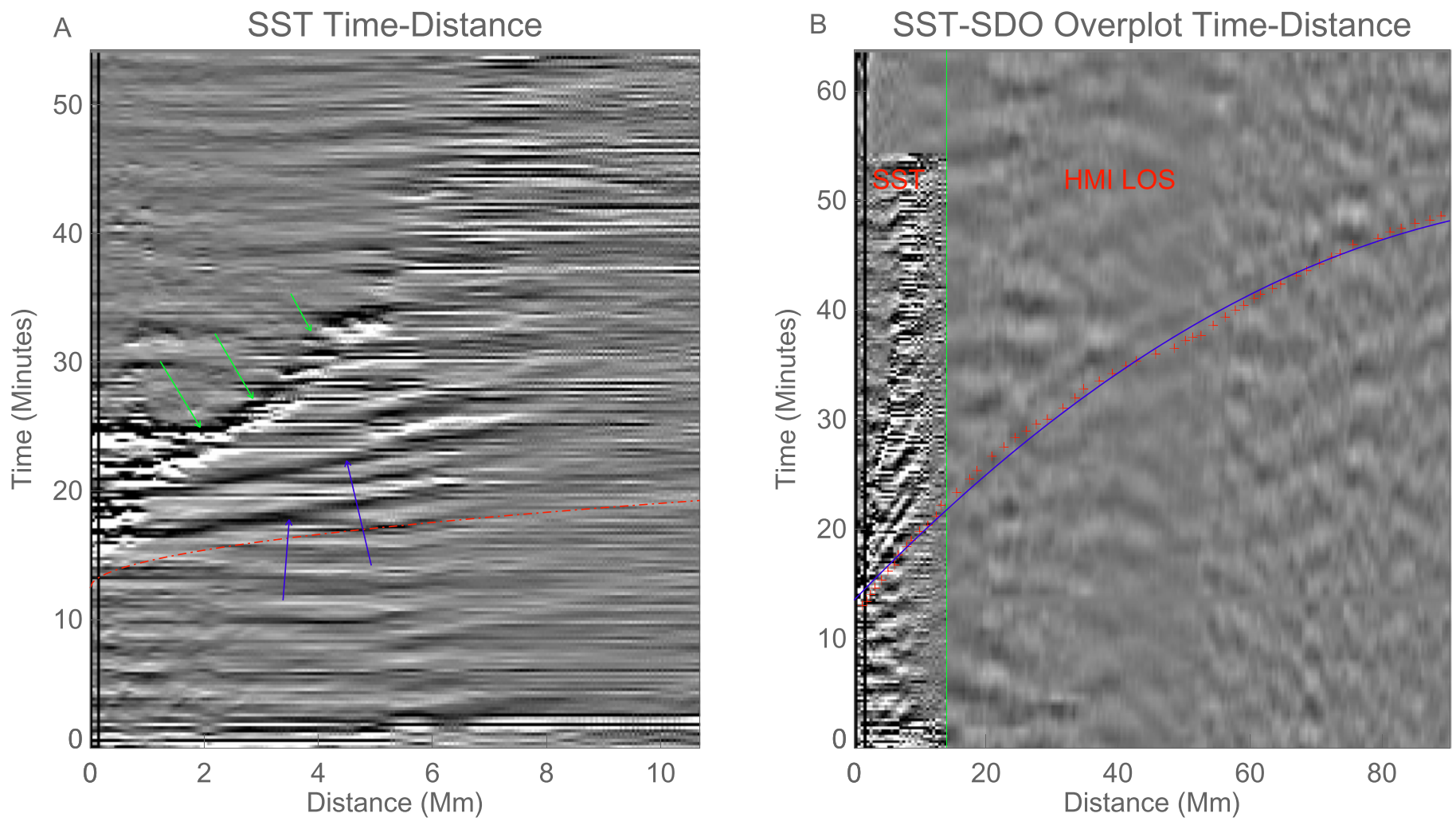

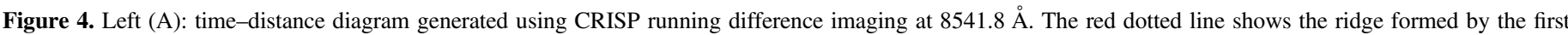

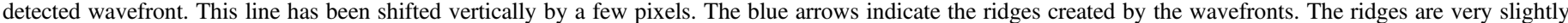

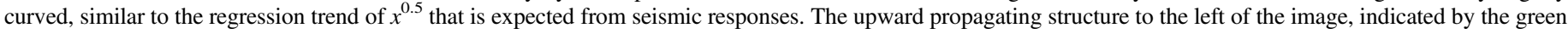

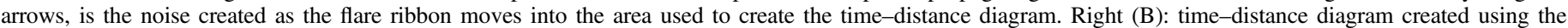

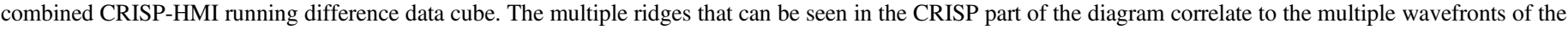

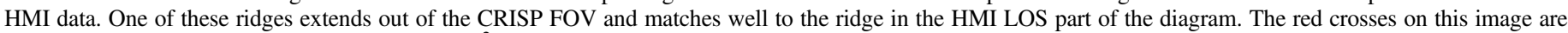

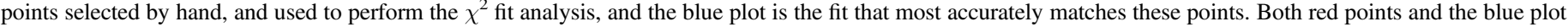
have been shifted vertically to ease the visibility of the ridge.

shown in the top left panel of Figure 2. The SQ is less pronounced in AIA $1600 \AA$. The AIA wavefronts are detected further away from the flare ribbon and appear later than in HMI. We investigated these AIA channels in an attempt to follow the upwards propagating response to the SQ, between the HMI LOS and the $8542 \AA$ observations. The AIA 1600 and $1700 \AA$ observations were the only channels that detected the response. These channels are sensitive to the upper photosphere and photosphere, respectively (Leman et al. 2012). We also investigated the AIA $304 \AA$ observations, which are sensitive to the high-chromosphere/transition region (Leman et al. 2012), to determine if the response was able to propagate to these heights; however, no such response was detected.

\subsection{LOS Velocity of the SQs}

We have also analyzed the spectral profiles cospatial to these apparent wavefronts using Ca II $8542 \AA$ data. The line profiles were investigated to determine the level of any line asymmetries. Each wavefront was subdivided into $20 \times 20$ pixel $^{2}$ areas for computational ease. A line profile was generated from each of the boxes, and the profiles were combined to create an average for each crest and trough. The same procedure was applied as the crests and troughs moved across the FOV. An average "quiet" profile was created from the same spatial location before it was crossed by the wavefront. The quiet profiles were averaged over 20 consecutive frames that did not show any intensity variations or velocity shifts to minimize the influence of the nearby sunspot.

In order to quantify the line asymmetries, we generated intensity ratios between the blue and red peaks. The ratios were created by integrating the line flux within $\pm 0.1 \AA$ of the maximum for the blue $\left(I_{B}\right)$ and the red $\left(I_{R}\right)$ peaks respectively. These peaks were found by fitting the line profiles with two single Gaussians, one for the red peak and one for the blue. The same procedure was applied to the quiet profiles. In Figure 5, asymmetries in the Ca II $8542 \AA$ line profiles created from crests and troughs are clearly present. The initial line profile was constructed from crests approximately $4 \mathrm{Mm}$ from the epicenter and the evolved profiles were sampled after the crest had propagated to $5 \mathrm{Mm}$ from the epicenter. These distances were selected as they were the best positions to find a wavefront that was significantly far from the ribbon, minimizing any intensity contamination. Any further from this chosen distance, the wavefronts tended to interfere with each other or the ribbon, making the creation of a single evolved wavefront line profile impossible. Poor seeing was also a constraint in regards to which frames were of a good enough quality to create a reliable line profile. Both the initial and evolved profiles displayed the same trend, with the crest showing a blue asymmetry and the trough showing a slight red asymmetry.

Normalizing the crests and troughs to their quiet profiles allowed any underlying asymmetries to be negated. Four sets of 

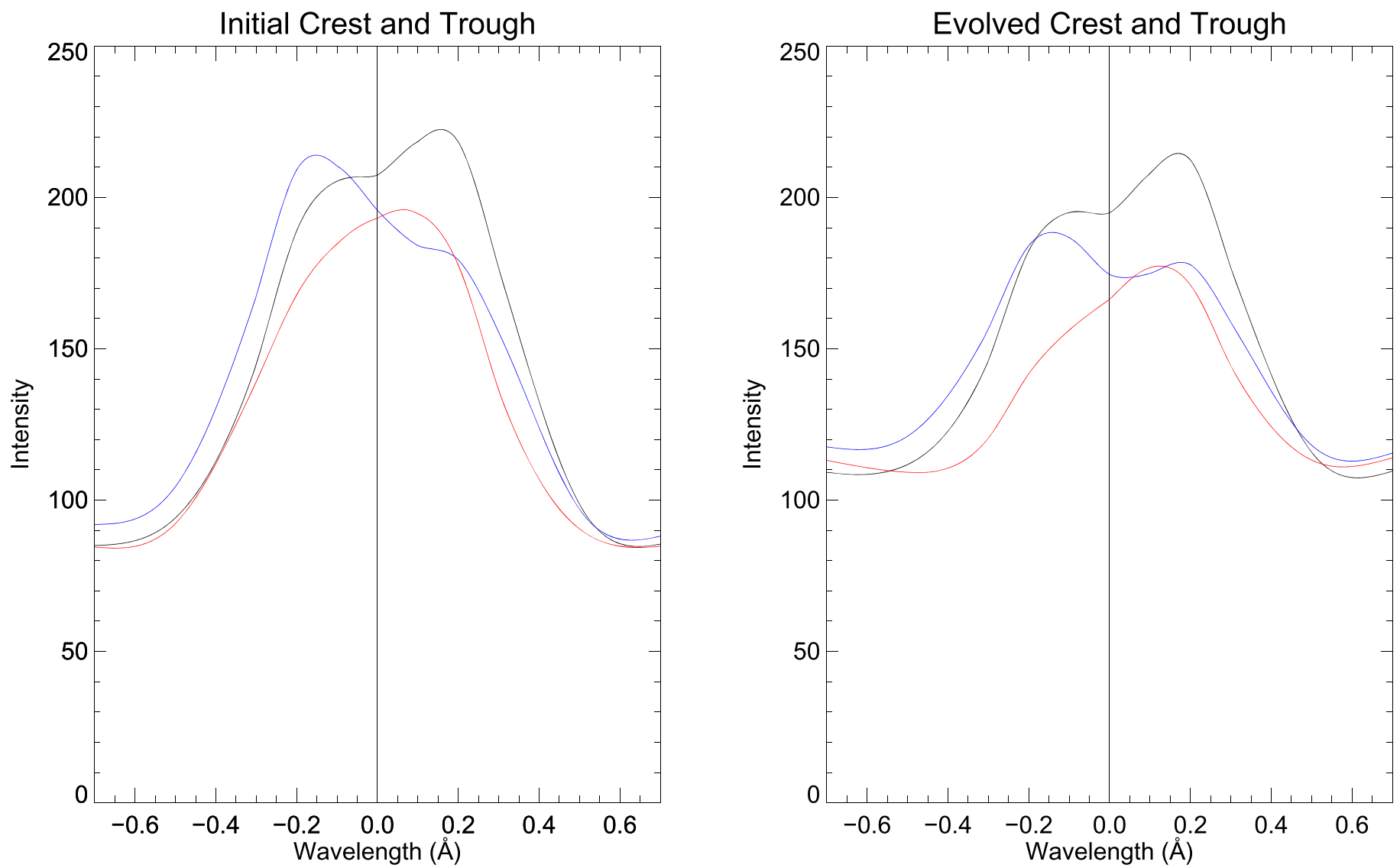

Figure 5. Example of the line profiles created for each wavefront. A total of four wavefronts were sampled. The "initial" line profiles are shown on the left and the "evolved" line profiles are shown on the right. Quiet line profiles are plotted in black whereas crests and troughs are shown in blue and red, respectively. The initial line profiles were taken early in this wavefront propagation, and the evolved profile was taken after the wavefront had time to move across the FOV.

crests and troughs were investigated, and the initial and evolved normalized asymmetry ratios are displayed in Table 1. Following normalization against their quiet profiles, the trough line profiles had an $I_{B} / I_{R}$ ratio of $\approx 1$. The trends shown by the line asymmetries provide evidence that the seismic response creates a velocity perturbation.

We used the NICOLE inversion algorithm to create an LOS velocity map of the propagating seismic response. NICOLE solves the multilevel NLTE radiation transfer problem for each pixel by following the preconditioning method set out in SocasNavarro \& Bueno (1997). It perturbs a number of parameters such as temperature, magnetic field, electron density, microturbulence, and LOS velocity of an initial atmosphere to find the best match with the observations (Socas-Navarro et al. 2000). The preinitial atmosphere that was used in this case is the Harvard-Smithsonian Reference Atmosphere model (Gingerich et al. 1971). The CRISP observations were interpolated to ensure even spacing between each spectral point with weighting assigned only to the observed spectral positions. A $2 \times 2$ binning was applied across the entire image. Each line profile was normalized with a number of reference profiles, taken from a "quiet" frame, across a multitude of regions with no major flare disturbances. The results of this step were used as the initial guess atmosphere for the subsequent inversions.

The data were then inverted to obtain an initial estimate of the atmosphere with very little vertical stratification. The inversion used four nodes in temperature, one node in LOS velocity, one node in microturbulence, and one node in LOS magnetic flux density. The output was smoothed spatially in
Table 1

Table of Normalized Asymmetry Ratios of $I_{B} / I_{R}$ for Each Line Profile

\begin{tabular}{ccccc}
\hline \hline & Initial Crest & $\begin{array}{c}\text { Initial } \\
\text { Trough }\end{array}$ & Evolved Crest & $\begin{array}{c}\text { Evolved } \\
\text { Trough }\end{array}$ \\
\hline Wave 1 & 1.22 & 0.99 & 1.25 & 0.95 \\
\hline Wave 2 & 1.22 & 1.00 & 1.22 & 1.04 \\
\hline Wave 3 & 1.24 & 0.94 & 1.23 & 1.06 \\
\hline Wave 4 & 1.18 & 1.05 & 1.18 & 0.99 \\
\hline
\end{tabular}

three dimensions, and this was used as input in the next iteration. This cycle involved seven nodes in temperature, three in LOS velocity, three in LOS magnetic flux density, one in transverse magnetic flux density, and one node in microturbulence. A third cycle was attempted, with increased nodes in both magnetic flux density and velocity, but this did not show significant improvement from the second cycle. Figure 6 shows the velocity maps created using NICOLE inversions for the entire FOV (left-hand panel) and for a zoomed region around the apparent wavefront. The wavefronts in the lower chromosphere at an optical depth of $\log \tau_{5000 \AA}=-3$ display clear upflows, which is to be expected given the CTTM of SQ generation.

The NICOLE outputs were compared with the COG method (Uitenbroek 2003) that can also be used to calculate Doppler velocities. Given that this is the first detection of the 

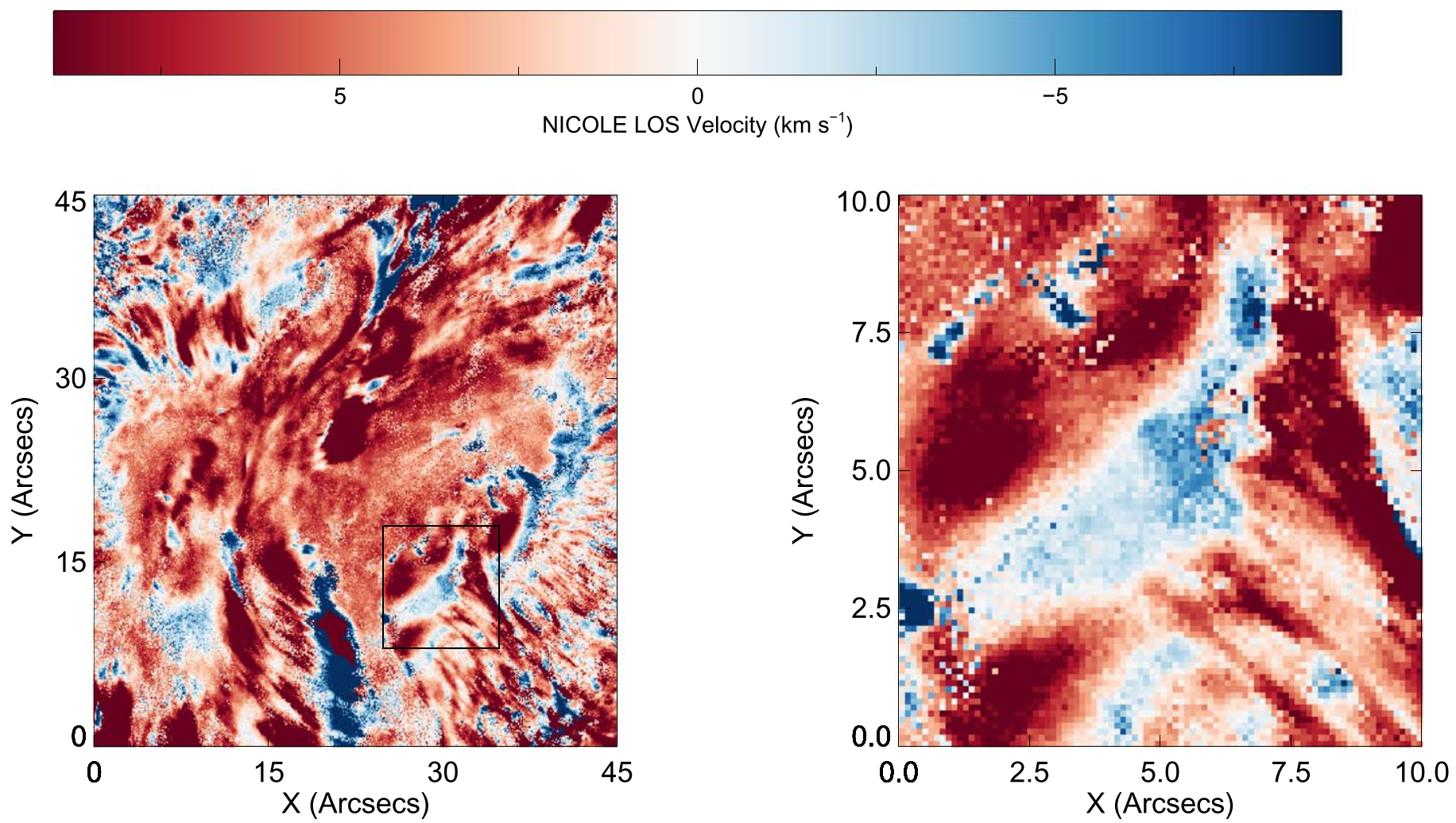

Figure 6. LOS velocity maps created using the NICOLE Inversion algorithm on the Ca II 8542 A data for the full FOV (left panel) and a zoomed in region around the wavefront (right panel). The black box indicates the FOV plotted in the right-hand panel. The NICOLE velocity map has been created for a log $\tau_{5000}=-3$.

chromosphere responding to an SQ, we wanted to estimate the corresponding LOS velocities with different, independent methods. The average LOS velocity of the Ca II $8542 \AA$ wave indicated in Figure 6 found using the COG method is $2.39 \mathrm{~km} \mathrm{~s}^{-1}$ while the corresponding velocity value determined from NICOLE is $3.20 \mathrm{~km} \mathrm{~s}^{-1}$. This difference can be accounted for by the simplicity of the COG method; however, it is below the velocity accuracy we can obtain with our spectral sampling. Therefore, we assume that the upflow velocity is approximately $3.2 \mathrm{~km} \mathrm{~s}^{-1}$, given that NICOLE is more intricate than the COG method, we give it a higher weighting. As a comparison, we also analyzed the HMI LOS velocity cospatial to the wavefronts. The photospheric LOS velocity was approximately $2 \mathrm{~km} \mathrm{~s}^{-1}$, meaning it is consistent with the chromospheric velocities.

\section{Conclusions}

We investigated the seismic responses in the solar photosphere and chromosphere generated by the X9.3 GOES class solar flare of 2017 September 06. The cospatial and cotemporal analysis of imaging and imaging spectroscopy obtained from HMI, AIA in the photosphere, and CRISP in the chromosphere allowed us to conclude that photospheric SQs do have signatures in the chromosphere. Numerous wavefronts are apparent in time-distance diagrams constructed from difference imaged CRISP data; however, only one wavefront is apparent in HMI LOS velocity maps. This wavefront matches well to one wavefront from CRISP. The apparent propagation of this wavefront's plane-of-sky velocity was calculated to be increasing from $4.5 \mathrm{~km} \mathrm{~s}^{-1}$ initially to $29.5 \mathrm{~km} \mathrm{~s}^{-1}$ after 46.5 minutes.
We have used the COG method and NICOLE inversions of the Ca II $8542 \AA$ line to construct LOS velocity maps. The LOS velocities of the upflowing material increase as they move through the atmosphere. The average upflow velocity detected in the photosphere is $2 \mathrm{~km} \mathrm{~s}^{-1}$, with this increasing to $3.2 \mathrm{~km} \mathrm{~s}^{-1}$ in the lower chromosphere.

Interestingly, upper-chromospheric observations using the AIA $304 \AA$ channel show no evidence of the SQ. This may mean the seismic response was unable to propagate to this height. However, this may also be due to the blooming effects due the saturation of the flare ribbon, which are most pronounced between 11:54:30 UT and 12:47:07 UT. The magnetic canopy may also be inhibiting the propagation of the wavefront higher into the atmosphere.

To the best of our knowledge this is the first detection of a flare generated SQ in the chromosphere. However, we have shown that the signal of such responses is very weak, and does not move far from the flare ribbon in the chromosphere. The lack of previous detections of such responses in the chromosphere is not due to the lack of a response being present, but due to low spectral and temporal resolutions in previous observational setups.

The Swedish $1 \mathrm{~m}$ Solar Telescope is operated on the island of La Palma by the Institute for Solar Physics of Stockholm University in the Spanish Observatorio del Roque de los Muchachos of the Instituto de Astrofsica de Canarias. The Institute for Solar Physics is supported by a grant for research infrastructures of national importance from the Swedish Research Council (registration No. 2017-00625). UK access to the Swedish $1 \mathrm{~m}$ Solar Telescope was funded by the Science and Technology Facilities Council (STFC) under grant No. 
ST/P007198/1. S.Q. acknowledges support from the Northern Ireland Department for the Economy for the award of a $\mathrm{PhD}$ studentship. A.R., M.M., C.J.N., and S.K.P. acknowledge support from STFC under grant No. ST/P000304/1.

Note added in proof. After the acceptance of this paper we were notified that the hydrogen Balmer alpha data for the same flare were submitted for publication to Astronomy and Astrophysics (Zharkov et al. 2019). The Zharkov et al. (2019) paper uses directional acoustic holography and time-distance diagram detection techniques for acoustic signals on the surface to detect three acoustic sources and link their locations with the footpoints of magnetic ropes formed just prior to the X9.3 flare event. Their hydrodynamic modeling suggests that one of the quakes could produce ripples above the photosphere and is therefore in agreement with our findings.

\section{ORCID iDs}

Sean Quinn (10) https://orcid.org/0000-0002-3227-2321

Aaron Reid (iD https://orcid.org/0000-0002-7695-4834

Mihalis Mathioudakis (1) https://orcid.org/0000-00027725-6296

S. Krishna Prasad (iD https://orcid.org/0000-0002-0735-4501

Sergei Zharkov (iD https://orcid.org/0000-0002-2348-3522

\section{References}

Chen, F., Ding, M., Chen, P., \& Harra, L. 2011, ApJL, 470, 116

Couvidat, S., Birch, A. C., Kosovichev, A. G., \& Zhao, J. 2004, ApJ, 607, 554 Couvidat, S., Schou, J., Shine, R., et al. 2011, SoPh, 275, 285

de la Cruz Rodríguez, J., Löfdahl, M., Sütterlin, P., Hillberg, T., \& Rouppe van der Voort, L. 2014, A\&A, 573, A40
Domingo, V., Fleck, B., \& Poland, A. I. 1995, SoPh, 162, 1

Donea, A. 2011, SSRv, 158, 451

Donea, A. C., Braun, D. C., \& Lindsey, C. 1999, ApJL, 513, L143

Donea, A. C., \& Lindsey, C. 2005, ApJL, 630, L168

Gingerich, O., Noyes, R. W., Kalkofen, W., \& Cuny, Y. 1971, SoPh, 18, 347 Kosovichev, A. G. 2006, SoPh, 238, 1

Kosovichev, A. G. 2007, ApJL, 670, L65

Kosovichev, A. G. 2011, LNP, 832, 3

Kosovichev, A. G. 2015, in Extraterrestrial Seismology, ed. V. C. H. Tong \& R. A. Garcia (Cambridge: Cambridge Univ. Press), 306

Kosovichev, A. G., \& Zharkova, V. V. 1995, Helioseimology, 2, 341

Kosovichev, A. G., \& Zharkova, V. V. 1998, Natur, 393, 317

Leman, J. R., Title, A. M., Akin, D. R., et al. 2012, SoPh, 275, 17

Lindsey, C., \& Donea, A. C. 2008, SoPh, 251, 627

Löfdahl, M. G. 2002, Proc. SPIE, 4792, 146

Matthews, S. A., Harra, L. K., Zharkov, S., \& Green, L. M. 2015, ApJ, 812, 35

Matthews, S. A., Zharkov, S., \& Zharkova, V. V. 2011, ApJ, 739, 71

Moreton, G. E., \& Ramsey, H. E. 1960, PASP, 72, 357

Pesnell, W. D., Thompson, B. J., \& Chamberlin, P. C. 2012, SoPh, 275, 3

Russell, A. J. B., Mooney, M. K., Leake, J. E., \& Huson, H. S. 2016, ApJ, 831,42

Scharmer, G. B., Bjelksjo, K., Korhonen, T., Lindberg, B., \& Petterson, B. 2003, Proc. SPIE, 4853, 341

Scharmer, G. B., Narayan, G., Hillberg, T., et al. 2008, ApJL, 689, L69

Scherrer, P. H., Bogart, R. S., Bush, R. I., et al. 1995, SoPh, 162, 129

Scherrer, P. H., Schou, J., Bush, R. I., et al. 2012, SoPh, 275, 207

Schou, J., Scherrer, P., Bush, R., et al. 2011, SoPh, 275, 229

Sharykin, I. N., \& Kosovichev, A. G. 2018, ApJ, 864, 86

Socas-Navarro, H., \& Bueno, J. T. 1997, ApJ, 490, 383

Socas-Navarro, H., Trujillo Bueno, J., \& Ruiz Cobo, B. 2000, ApJ, 530, 977 Uitenbroek, H. 2003, ApJ, 592, 1225

Van Noort, M., Van Der Voort, L. R., \& Löfdahl, M. G. 2005, SoPh, 228, 191

Wolff, C. L. 1972, ApJL, 176, 833

Zhao, J., \& Chen, R. 2018, ApJL, 860, L29

Zharkov, S., Zharkova, V., Matthews, S., et al. 2019, A\&A, submitted

Zharkov, S. I., Green, L. M., Matthews, S. A., \& Zharkova, V. V. 2011, ApJL, 741.2, L35

Zharkova, V. V., \& Zharkov, S. I. 2007, ApJL, 664, 573 